\title{
Ba II 4554 Å speckle imaging as solar Doppler diagnostic
}

\author{
P. Sütterlin ${ }^{1}$, R. J. Rutten ${ }^{1}$, and V. I. Skomorovsky ${ }^{2}$ \\ 1 Sterrekundig Instituut Utrecht, Postbus 80000 , 3508 TA Utrecht, The Netherlands \\ e-mail: R.J.Rutten@astro.uu.nl \\ 2 Institute of Solar-Terrestrial Physics, 664033 Irkutsk, PO Box 4026, Russia \\ e-mail: skoal@iszf.irk.ru
}

Received 12 July 2001 / Accepted 20 August 2001

\begin{abstract}
We present observations testing the Dopplergram capability of a narrow-band ( $80 \mathrm{~m} \AA$ ) Lyot filter imaging the solar surface in the wings of the Ba II $4554 \AA$ resonance line in combination with speckle reconstruction to obtain high angular resolution. The Ba II line is found to be an excellent tool for high-resolution Doppler mapping thanks to opacity insensitivity to temperature variations and line-width insensitivity to thermal broadening. The resulting Dopplergrams show concentrated downflows of $1.2-2.2 \mathrm{~km} \mathrm{~s}^{-1}$ in intergranular lanes that probably mark magnetic fluxtubes. Two-wavelength profile sampling is found to suffice for high-resolution Dopplergram construction. The filter will be installed as part of a multi-wavelength speckle imaging system on the new Dutch Open Telescope.
\end{abstract}

Key words. Sun: atmosphere - Sun: photosphere - Sun: faculae, plages - techniques: image processing instrumentation: miscellaneous

\section{Introduction}

There are different ways to obtain Dopplergrams (radial velocity maps) of structures in the solar photosphere with high angular resolution, each having advantages and disadvantages. Spectrographs yield full line-profile information for precise Dopplershift evaluation but require spatial scanning by the spectrograph slit to derive two-dimensional maps, implying loss of simultaneity across the solar scene and large sensitivity to seeing variations. Filtergraphs obtain full-field images per exposure but only for one spectral passband at a time, while at least two are needed to derive Dopplergrams through subtraction which is also very sensitive to seeing variations. Fabry-Perot interferometers permit rapid passband tuning across line profiles and presently present, in combination with image stabilization systems, the most popular solution for two-dimensional solar Doppler mapping (e.g. Bonaccini et al. 1989; Kneer 1997; Kentischer 1998), partly with application of deconvolution techniques (Hirzberger 2001). Subtractive multi-exit spectrometry (MSDP, Mein 1977, 1991) is a hybrid compromise between spectral and spatial resolution, trading most of one spatial dimension against multiple spectrograph passbands within a sufficiently wide spectral line. Fiber reformatting of a two-dimensional input field to a linear

Send offprint requests to: P. Sütterlin,

e-mail: P.Suetterlin@astro.uu.nl spectrograph slit seems attractive (Kucera 1999) but suffers from focal ratio degradation (Rutten 1999). The advent of adaptive optics on optical solar telescopes will enable both spectrometer slit scanning and Fabry-Perot passband scanning with real-time correction of seeing degradation, so that the solar change time across the angular resolution element becomes the main limitation.

This paper describes a test which combines the principal alternative to adaptive optics, namely post-detection speckle reconstruction, with a tunable Lyot filter selecting narrow passbands in a specific line, namely the Ba II resonance line at $\lambda=4554 \AA$. The test was done in the context of developing focal-plane instrumentation for the new Dutch Open Telescope (DOT) on La Palma (Hammerschlag \& Bettonvil 1998; Rutten et al. 2001a). The DOT is presently being equipped with an advanced multi-wavelength imaging system (Sütterlin et al. 2001) that exploits the well-established speckle-masking image reconstruction technique (Weigelt \& Wirnitzer 1983; Lohmann et al. 1983; von der Lühe 1993) for large-volume tomography of the solar atmosphere (Rutten et al. 2001b). The test described here evaluates Dopplergram extension to this system.

A major advantage of speckle imaging, which applies to sub-meter class telescopes such as the $45-\mathrm{cm}$ aperture DOT, is that the full field is corrected for seeing degradation, not just the central isoplanatic patch. The field extent is therefore limited only by the camera 


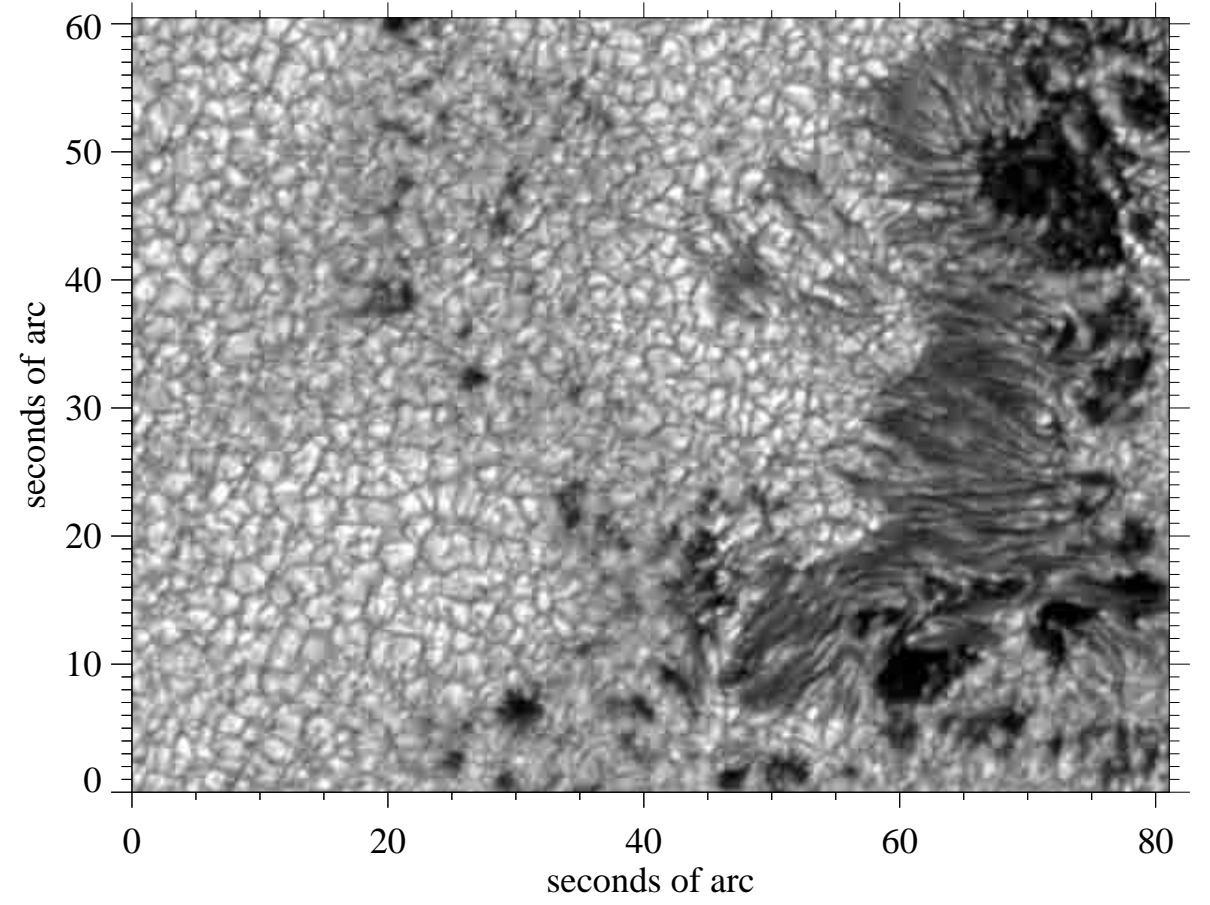

Fig. 1. Field of view for the Ba II $4554 \AA$ filter test. Intensity image constructed by summing the outer red and blue wing images in Fig. 4.

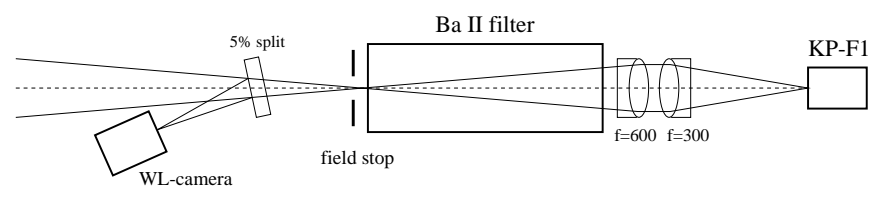

Fig. 2. Optical setup.

chip. Combination of speckle reconstruction with narrowband filter imaging enables the construction of full-field diffraction-limited Dopplergrams if sufficient specklegrams can be obtained within the solar change time.

A major advantage of the BaII resonance line is that barium is a very heavy atom, making the thermal contribution to the Dopplerwidth small. At high angular resolution much of the solar fine structure that used to be parametrized as "non-thermal microturbulence" in classical plane-parallel line formation modeling does no longer act as line broadening but is actually resolved, making the non-thermal broadening contribution small as well. The resulting steepness of the line wings brings large Doppler sensitivity. Fortunately, the Ba II $4554 \AA$ A line does not get too narrow to accommodate a Lyot filter passband because it is split into fourteen isotope and hyperfine structure components (Rutten 1978). In the spatiallyaveraged solar spectrum the line has a wide asymmetric core (Fig. 3). In highly resolved observations the line wings steepen while the core retains its width.

An additional advantage of the BaII $4554 \AA$ line is that it is, like its atomic twin CaII $\mathrm{K}$, a resonance line from the ground state of the dominant ionization stage. The line strength is therefore insensitive to the radiative overionization which awkwardly affects the opacity of most atomic lines in the optical solar spectrum. This makes interpretation relatively straightforward. In standard modeling the core is formed at height $h=700 \mathrm{~km}$

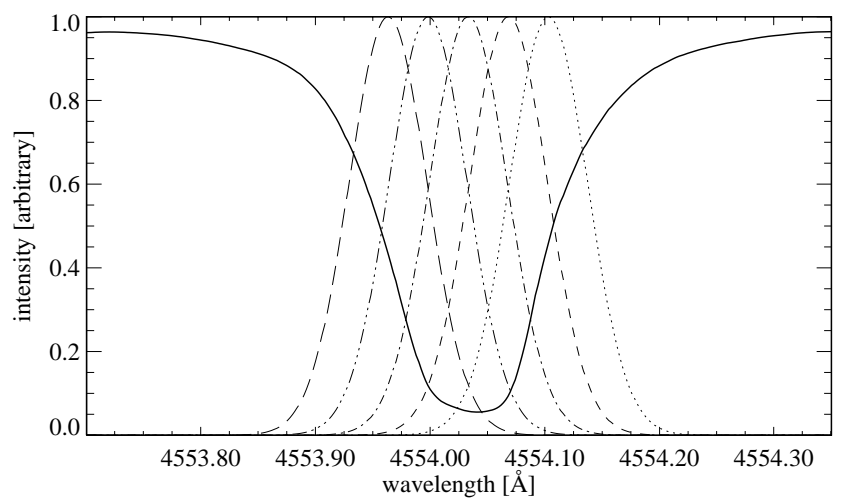

Fig. 3. Spatially-averaged disk-center profile of the Ba II $4554 \AA$ resonance line taken from Neckel (1999). The five tunable-filter bandpasses used in this paper are superimposed assuming a Gaussian bandpass shape.

with temperature sensitivity to the temperature minimum region (with lower temperature producing a deeper line, Sykorski \& Merkulenko 1988). The wings are photospheric. The line has unusually high coherency in its inner wing scattering (Rutten 1978; Rutten \& Milkey 1979; Uitenbroek \& Bruls 1992) but this affects its formation only when observed close to the solar limb where the line has the highest linear polarization of the entire optical spectrum (J. Trujillo Bueno, private communication; Wiehr 1981). The main isotopic component has effective Landé factor $g_{\text {eff }}=1.16$ and may be suited to Stokes polarimetry.

A narrow-band tunable birefringent Lyot filter to observe the sun in the BaII $4554 \AA$ line was built and used at the Irkutsk Institute of Solar-Terrestrial Physics in the early 1970's by the third author and coworkers (Aleksandrovich et al. 1975; Skomorovsky et al. 1976) on the premise that this line resembles CaII K in every 

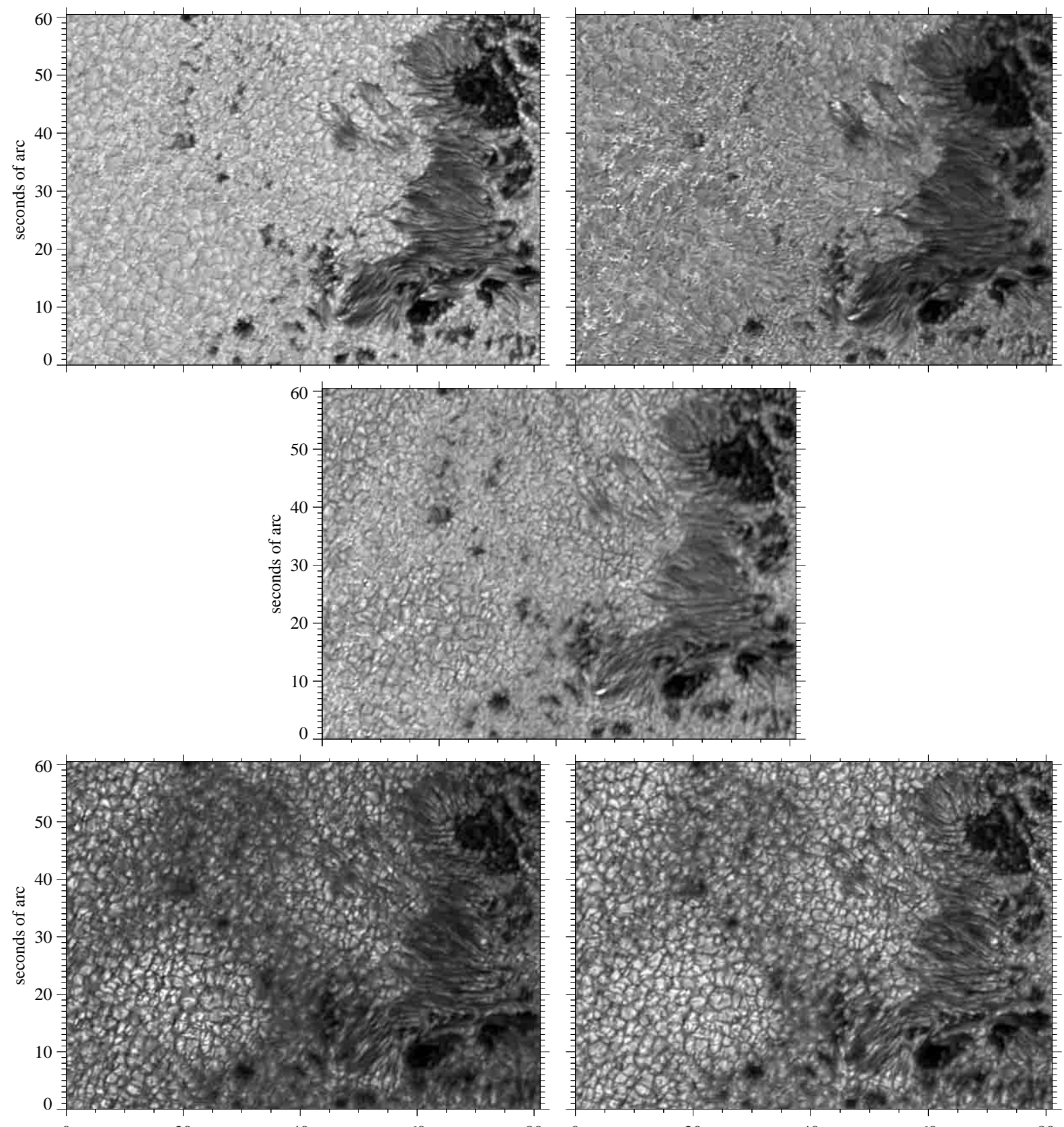

0

20

seconds of arc

60

$80 \quad 0$

20

40

seconds of arc

60

80

Fig. 4. Five filtergrams constituting one scan through the BaII $4554 \AA$ line. The wavelength settings are $\Delta \lambda=$ $-70,-35,0,+35,+70 \mathrm{~m} \AA$ from line center as indicated in Fig. 3.

respect except elemental abundance so that it might be as useful a diagnostic of the upper photosphere as CaII $\mathrm{K}$ is of the chromosphere. The filter has an extremely narrow bandwidth of only $80 \mathrm{~m} \AA$ and can also be tuned to $\mathrm{H} \beta$.

\section{Observations}

The observations were done on July 15, 2000 with the Swedish Vacuum Solar Telescope (SVST) on La Palma during its last observing season. The DOT CCD camera (Hitachi KP-F1, $768 \times 572$ px) and data acquisition system (8-bit frame grabber running at 8 frames/s) were used instead of the slower Swedish telescope Megaplus camera system in order to obtain sufficient speckle frames with sufficient $\mathrm{S} / \mathrm{N}$ per speckle burst. Figure 2 depicts the optical setup: the BaII filter was put immediately after the prime focus. With the long focal length of the SVST $(22 \mathrm{~m})$ this is a close-to-telecentric configuration. A 2:1 re-imaging adapted the image scale to the pixel size of the camera $(8.3 \mu)$ and increased the light level, improving $\mathrm{S} / \mathrm{N}$. The image scale (measured with a calibrated target in the prime focus) was $0.145 / \mathrm{px}$, so that the diffraction limit of the $48 \mathrm{~cm}$ telescope $\left(0^{\prime \prime} 2\right)$ was not fully sampled.

The Irkutsk filter was manually tuned to the five wavelengths covering the BaII line at $35 \mathrm{~m} \AA$ intervals as 


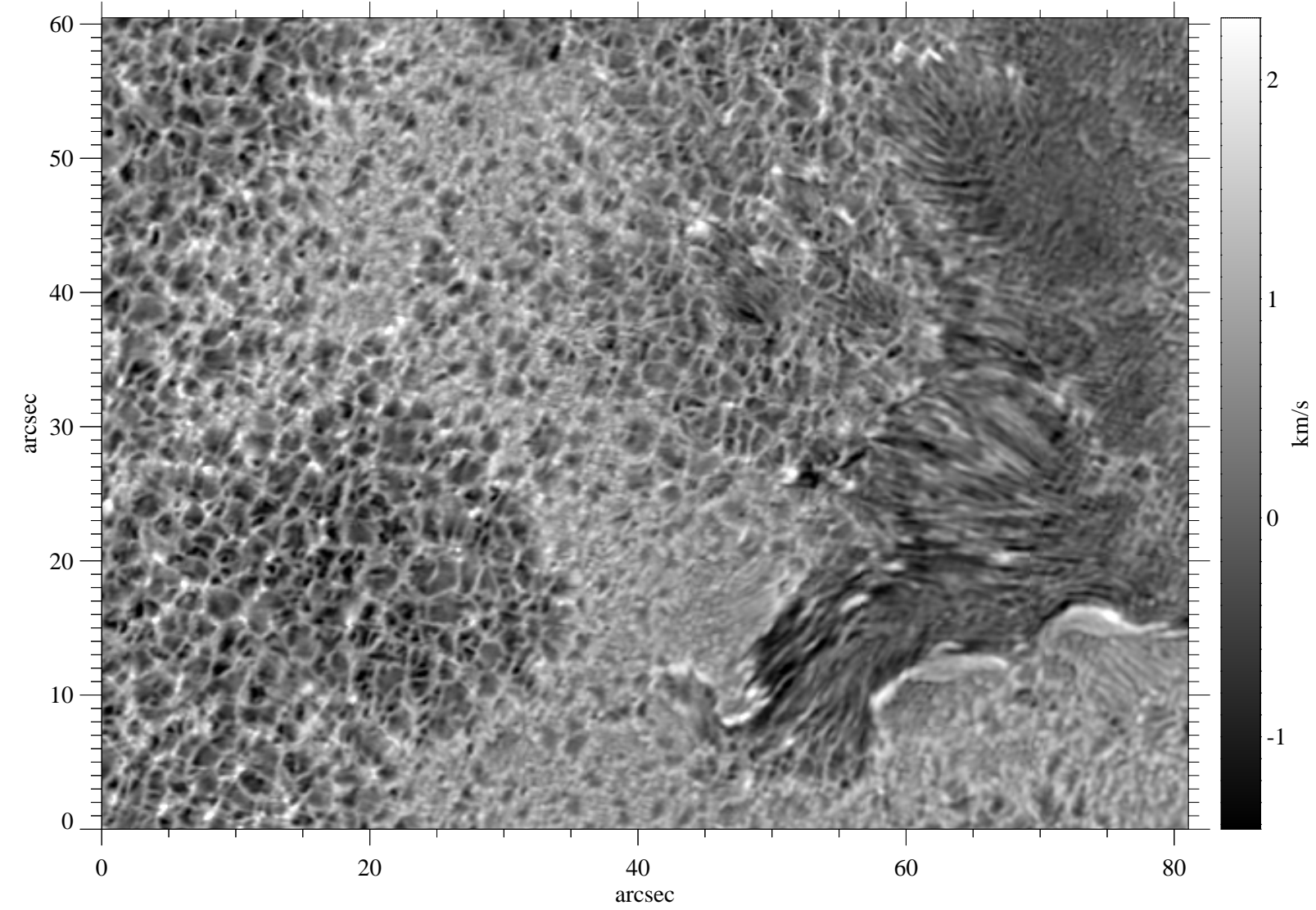

Fig. 5. Ba II $4554 \AA$ Dopplergram. The bar at right specifies the greyscale coding, with positive velocities denoting redshift and zero velocity defined by the line-center position of the average profile determined from the flatfield images taken at disk center. The area at the lower left contains normal granulation but also shows many small-area downdrafts (bright) located in intergranular lane junctions which probably mark magnetic fluxtubes. The lighter band through the middle shows that the plage with abnormal granulation has average downdraft. The striated area on the right shows that the active region contains flows along field-aligned structures in alternate directions.

indicated in Fig. 3. At each wavelength setting a speckle burst of 100 frames was taken within $12 \mathrm{~s}$, well within the solar change time $\left(30 \mathrm{~s}\right.$ per $0^{\prime \prime} .3$ resolution at $7 \mathrm{~km} \mathrm{~s}^{-1}$ sound speed). The exposure per speckle frame was $20 \mathrm{~ms}$, sufficiently short to freeze atmospheric seeing but yet yielding sufficient $\mathrm{S} / \mathrm{N}$ for speckle processing even with the $80 \mathrm{~m} \AA$ filter bandpass. A full scan covering the line with five sequential wavelength settings took approximately $130 \mathrm{~s}$. Thirteen such scans were obtained during a 25-min period of fair-to-good seeing (Fried parameter $\left.r_{0}=3-10.6 \mathrm{~cm}\right)$.

The target was a part of active region AR 9077 at viewing angle $\cos \vartheta=0.62$. The field of view is shown in Fig. 1. It contains sunspots with much highly-structured penumbra in the righthand part, plage with small pores at center, and more or less quiet sun at left. The network boundaries in the lefthand part and the plage area at center contain numerous tiny bright points within the intergranular lanes. There are also areas of undisturbed granulation in the lefthand part.

\section{Data reduction}

The speckle bursts were processed by the speckle masking method (Weigelt \& Wirnitzer 1983; von der Lühe 1984) using the Göttingen reconstruction code (de Boer 1996; Sütterlin \& Wiehr 2000), producing one diffractionlimited image from each burst that is free of atmospheric disturbances. Each wavelength position so yielded a 25 -min time sequence.

Since each five-wavelength scan through the line took about $130 \mathrm{~s}$, the filtergrams of one scan cannot be used directly to derive Doppler velocities because the solar five-minute oscillation pattern changed in the meantime. Each of the five time sequences per wavelength position were therefore Fourier-filtered using a cone filter in $(k, \omega)$ space. The resulting filtered data cubes were then spatially aligned, resulting in a fourdimensional hypercube with two spatial axes, one wavelength axis, and one time axis.

\section{Results}

Figure 4 shows the speckle-reconstructed images resulting from one five-wavelength scan. The filtergrams in the two line wings are strikingly different. The last image shows granulation very pronouncedly, whereas it nearly vanishes in the second (upper right) image which displays many bright points of much smaller size. The active region at right also appears very differently in the 


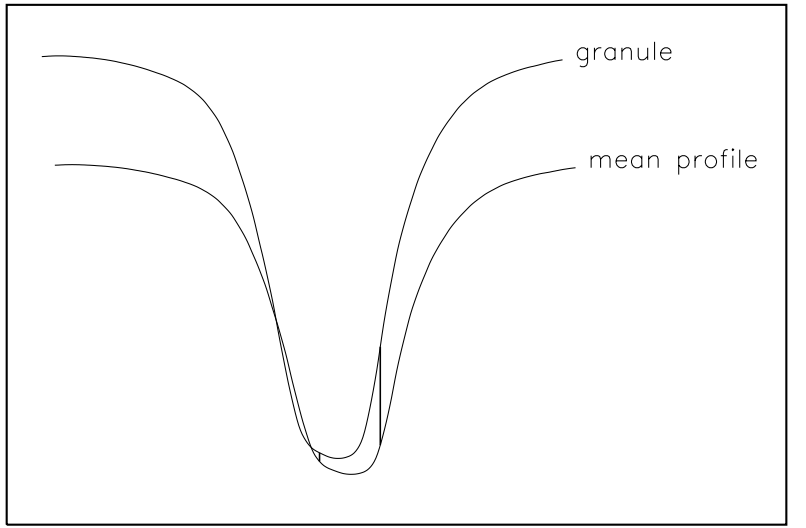

Fig. 6. Schematic illustration of the influence of correlation between intensity and line-of-sight velocity on the observed contrast in a narrow-band filtergram. The mean profile is the disk-center Ba II $4554 \AA$ line from Fig. 3. To mimic a granule it is multiplied by 1.3 and blueshifted over $1 \mathrm{~km} \mathrm{~s}^{-1}$. Such bright blueshifted structures show high contrast in the red wing and small contrast in the blue wing. In this case the red-wing contrast indicated by the vertical bar at right is $I_{\text {gran }} / I_{\text {mean }}=$ 2.2 whereas the blue-wing contrast sampled at left is only 1.13 . The pattern reverses when bright structures are redshifted.

two line wings. These large differences result from specific intensity-velocity correlations as illustrated in Fig. 6. In the red line wing the intensity contrast is much enhanced for structures with negative intensity-velocity correlation $(\delta I \cdot \delta v<0)$, such as solar granulation in which bright granules move upwards. Reversely, the apparent brightness contrast is smaller for structures with positive $\delta I \cdot \delta v$. In the blue wing the sign reverses so that the apparent contrast of bright structures harboring downward flows is enhanced. Thus, the bottom-right image in Fig. 4 enhances the granulation but suppresses bright intergranular points with downdrafts. The latter are emphasized in the upper-right image.

Figure 5 shows an example of a Dopplershift map that was computed by determining first moments of parabolic fits to the five cone-filtered wavelength samples in one profile scan at every spatial position. Redshift is chosen positive so that downdrafts appear bright, a choice which emphasizes downdraft topology. The resulting Dopplergram is unusually rich in fine structure. The area with normal granulation at the lower left shows not only the downdraft network pattern made by the intergranular lanes, but also numerous enhancements marking small-scale downdrafts located within the lanes. They are also seen as small bright features in the second panel of Fig. 4; the Dopplergram establishes that they reside in intergranular lanes, preferentially in lane junctions.

The area with abnormal granulation in the middle of the Dopplergram shows a general brightness enhancement, implying overall average downdraft - it is markedly dark in similar fashion in the inner red wing image in the lowerleft panel of Fig. 4. The larger density of small downdrafts in this area is seen well in the upper-right panel of Fig. 4.

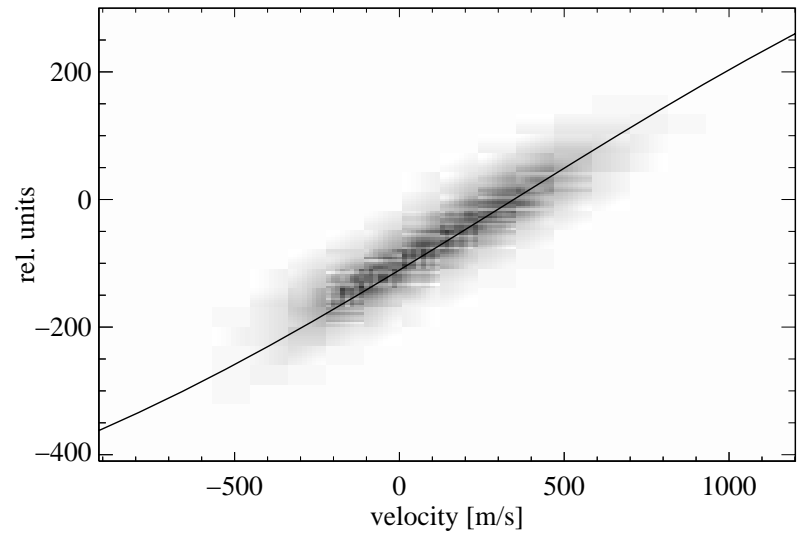

Fig. 7. Scatter plot of two-point intensity differences as defined by Eq. (1) (vertical) against five-wavelength Dopplershift measurement (horizontal) per pixel. The curve shows the relation expected from the mean disk-center profile and the bandpass shape shown in Fig. 3.

The pores in the plage area generally have adjacent high-speed downdrafts at small sites in their perimeter. The pores themselves contain lower-speed downdrafts in complex patterns.

The active region in the righthand part of Fig. 5 shows much filamentary contrast, indicating the presence of bidirectional mottle flows in alternating arrangement.

\section{Discussion}

The small bright specks in the intergranular lanes in Fig. 5 mark high-speed downdrafts ranging between 1.0 and $2.2 \mathrm{~km} \mathrm{~s}^{-1}$. Their average diameter is only $0^{\prime \prime} 34$, not much larger than the resolution limit of our data. They often correspond to intergranular bright points in the intensity image in Fig. 1, but the latter contains also intergranular bright points without marked downdraft and some downdraft points correspond to dark points in the intergranular lanes.

Intergranular bright points are often called "network bright points" in the literature and become visible at sufficiently high angular resolution, in particular in the Fraunhofer $G$ band around $\lambda=4305 \AA$ (e.g. Muller 1994; Title \& Berger 1996), for example on recent DOT movies available at http://dot.astro.uu.nl. They mark magnetic concentrations that are believed to be the observational counterpart to the modeler's fluxtubes. In numerical simulations and also in empirical magnetostatic modeling from Stokes profiles, these fluxtubes harbor fast downdrafts in their immediate surroundings (e.g. Steiner et al. 1998). It may be that the intergranular downdrafts seen here mark such steady downflows adjacent to quasi-static fluxtubes. It is also possible that they mark more dynamical downdrafts as may occur in convective collapse. The fact that some of the intergranular bright points in Fig. 1 coincide with large downdraft in the Doppler map in Fig. 5 while other bright points do not calls for combination of high-resolution Doppler maps as presented here with simultaneous and cospatial high-resolution $G$-band imaging in long-duration time sequences. 
With respect to measurement technique it is of interest to add that we have also produced Dopplershift maps using only the pair of inner red and blue line-wing images by evaluating the ratio

$V=\frac{I_{\text {left }}-I_{\text {right }}}{I_{\text {left }}+I_{\text {right }}}$

for every spatial pixel. Dopplershift determinations with such simple algorithms often meet problems caused by seeing changes, insufficient angular resolution, or intrinsic spatial variation of the line-profile shape. We find that using the BaII $4554 \AA$ line with its largely temperatureinsensitive profile and with our spatial resolution of $0^{\prime \prime} 3$ and consistent removal of temporal seeing variations, the two methods produce essentially identical results. Figure 7 quantifies this good correspondence in the form of a scatter plot comparing the two evaluations per pixel, and adds a theoretical curve computed from the disk-center atlas profile on the assumption of Gaussian-shaped filter transmission at the nominal $80 \mathrm{~m} \AA$ bandpass halfwidth. The empirical scatter cloud and the prediction correspond very well, implying that two-wavelength sampling suffices for Ba II $4554 \AA$ A Dopplergram construction. This is a welcome finding since less wavelength sampling permits faster Dopplergram acquisition cadence.

\section{Conclusion}

Our test demonstrates that the BaII $4554 \AA$ line offers an excellent diagnostic to measure Doppler velocities from narrow-band filtergrams combined with speckle restoration. The quality and resolution of the resulting Dopplergrams, as illustrated in Fig. 5, is so high that permanent installation of the Irkutsk filter in the new DOT secondary optics system is a desirable option. An accommodation study has shown that, even though the filter is large and heavy, it can be mounted at the top of the DOT using a telecentric re-imaging configuration that maintains optical quality at the diffraction limit at the $4554 \AA$ wavelength. The present aim is to implement Ba II Doppler mapping as a standard part of multi-channel high-resolution observing with the DOT, including simultaneous $G$-band imaging. A high-resolution polarimetry test of this line will also be of interest.

Acknowledgements. We are indebted to A. Ludmany for the initial suggestion to try the Irkutsk filter at La Palma, to G. B. Scharmer for allocating SVST telescope time in ESMN context, and to F. C. M. Bettonvil, R. H. Hammerschlag and G. N. Domishev for assistance with the observations. The SVST is operated by the Royal Swedish Academy of Sciences at the Spanish Observatorio del Roque de los Muchachos of the Instituto de Astrofísica de Canarias. P. Sütterlin's research is funded by the European Solar Magnetometry Network (ESMN) under EC-TMR contract ERBFMRXCT98019. Improvement of the barium filter was funded by the Russian Foundation for Basic Researches (grant 00-02-16068-a). The travel of V. I. Skomorovsky and G. N. Domishev to La Palma was funded by SOZOU, NOVA and LKBF. DOT research is funded by NWO, NOVA and Utrecht University.

\section{References}

Aleksandrovich, S., Domishev, G., Korovkin, A., \& Skomorovsky, V. 1975, Novaja technika v astronomii, 5,34

Bonaccini, D., Righini, A., Cavallini, F., \& Ceppatelli, G. 1989, A\&A, 217, 368

de Boer, C. R. 1996, A\&AS, 120, 195

Hammerschlag, R. H., \& Bettonvil, F. C. M. 1998, New Astron. Rev., 42, 485

Hirzberger, J., Koschinsky, M., Kneer, F., \& Ritter, C. 2001, A\&A, 367, 1011

Kentischer, T. J., Schmidt, W., Sigwarth, M., \& Uexküll, M. V. 1998, A\&A, 340, 569

Kneer, F. 1997, in ed. B. Schmieder, J. C. del Toro Iniesta, \& M. Vázquez, Advances in the Physics of Sunspots, Procs. First Adv. in Solar Physics Euroconf., ASP Conf. Ser., 116, 329

Kucera, A., Rybák, J., Wöhl, H., \& Hanslmeier, A. 1999, in Third Advances in Solar Physics Euroconference: Magnetic Fields and Oscillations, ASP Conf. Ser., 184, 319

Lohmann, A. W., Weigelt, G., \& Wirnitzer, B. 1983, Appl. Opt., 22, 4028

Mein, P. 1977, Solar Phys., 54, 45

Mein, P. 1991, A\&A, 248, 669

Muller, R. 1994, in ed. R. J. Rutten, \& C. J. Schrijver, Solar Surface Magnetism, NATO ASI Ser. C 433 (Kluwer, Dordrecht), 55

Neckel, H. 1999, Solar Phys., 184, 421

Rutten, R. J. 1978, Solar Phys., 56, 237

Rutten, R. J. 1999, in ed. T. R. Rimmele, K. S. Balasubramaniam, \& R. R. Radick, High Resolution Solar Physics: Theory, Observations, and Techniques, Procs. 19th NSO/Sacramento Peak Summer Workshop, ASP Conf. Ser., 183, 296

Rutten, R. J., Hammerschlag, R. H., Sütterlin, P., \& Bettonvil, F. C. M. 2001a, in Advanced Solar Polarimetry - Theory, Observation, and Instrumentation, Procs. 20th NSO/SP Summer Workshop, ed. M. Sigwarth, ASP Conf. Ser., 236, 25

Rutten, R. J., Hammerschlag, R. H., Sütterlin, P., Bettonvil, F. C. M., \& van der Zalm, E. B. J. 2001b, in The Solar Cycle and Terrestrial Climate, Procs. 1st Solar \& Space Weather Euroconference, ed. A. Wilson, ESA Special Publication SP-463, Estec, Noordwijk, 611

Rutten, R. J., \& Milkey, R. W. 1979, ApJ, 231, 277

Skomorovsky, V., Merkulenko, V., \& Poliakov, V. 1976 Solnechnye dannye, 5,73

Steiner, O., Grossmann-Doerth, U., Knölker, M., \& Schüssler, M. 1998, ApJ, 495, 468

Sütterlin, P., Hammerschlag, R. H., Bettonvil, F. C. M., et al. 2001, in Advanced Solar Polarimetry - Theory, Observation, and Instrumentation, Procs. 20th NSO/SP Summer Workshop, ed. M. Sigwarth, ASP Conf. Ser., 236, 431

Sütterlin, P., \& Wiehr, E. 2000, Solar Phys., 194, 35

Sykorski, J., \& Merkulenko, V. E. 1988, Issledovaniia Geomagnetizmu Aeronomii i Fizike Solntsa, 83, 69

Title, A. M., \& Berger, T. E. 1996, ApJ, 463, 797

Uitenbroek, H., \& Bruls, J. H. M. J. 1992, A\&A, 265, 268

von der Lühe, O. 1984, J. Opt. Soc. Am. A, 1, 510

von der Lühe, O. 1993, A\&A, 268, 374

Weigelt, G., \& Wirnitzer, B. 1983, Opt. Lett., 8, 389

Wiehr, E. 1981, A\&A, 95, 54 